\title{
Correction to: Minimal Models I
}

\section{Philippe Di Francesco, Pierre Mathieu and David Sénéchal}

\section{Correction to: P. Di Francesco et al., Minimal Models I, \\ DOI 10.1007/978-1-4612-2256-9_7}

The book was inadvertently published with missing figure 7.1 on page 207 . This error has now been corrected with this correction.
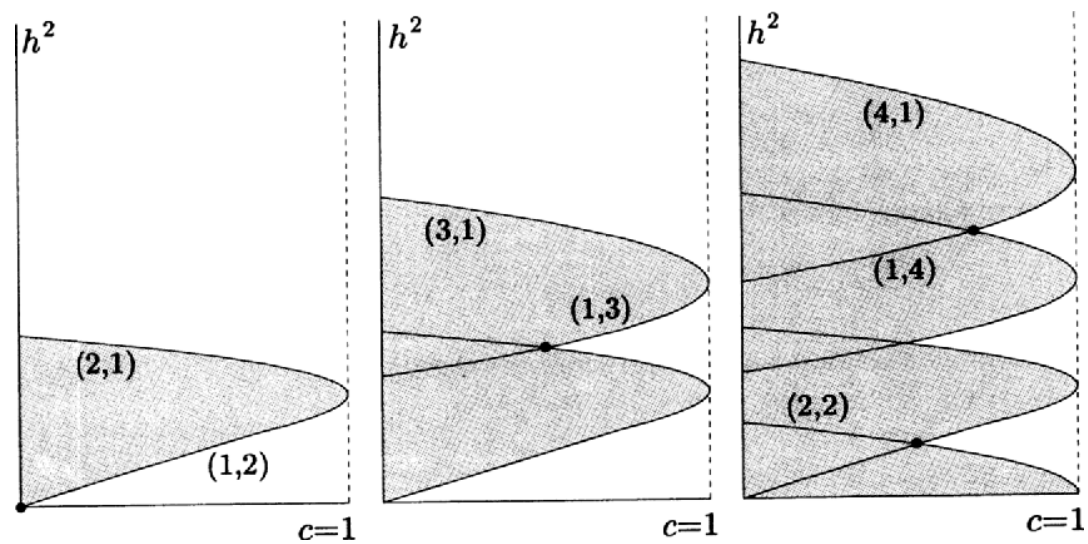

The updated online version of this chapter can be found at https://doi.org/10.1007/978-1-4612-2256-9_7 